\title{
A new lower bound on the independence number of a graph and applications
}

\author{
Michael A. Henning \\ Department of Mathematics \\ University of Johannesburg \\ Auckland Park, 2006 South Africa \\ mahenning@uj.ac.za \\ Justin Southey \\ Department of Mathematics \\ University of Johannesburg \\ Auckland Park, 2006 South Africa \\ just.so@presentable.me
}

\author{
Christian Löwenstein \\ Institute of Optimization and Operations Research \\ Ulm University \\ Ulm 89081, Germany \\ christian.loewenstein@uni-ulm.de \\ Anders Yeo \\ Engineering Systems and Design \\ Singapore University of Technology and Design \\ 20 Dover Drive Singapore, 138682, Singapore \\ and \\ Department of Mathematics \\ University of Johannesburg \\ Auckland Park, 2006 South Africa \\ andersyeo@gmail.com
}

Submitted: Jul 25, 2013; Accepted: Feb 11, 2014; Published: Feb 21, 2014

Mathematics Subject Classifications: 05C65

\begin{abstract}
The independence number of a graph $G$, denoted $\alpha(G)$, is the maximum cardinality of an independent set of vertices in $G$. The independence number is one of the most fundamental and well-studied graph parameters. In this paper, we strengthen a result of Fajtlowicz [Combinatorica 4 (1984), 35-38] on the independence of a graph given its maximum degree and maximum clique size. As a consequence of our result we give bounds on the independence number and transversal number of 6 -uniform hypergraphs with maximum degree three. This gives support for a conjecture due to Tuza and Vestergaard [Discussiones Math. Graph Theory 22 (2002), 199-210] that if $H$ is a 3-regular 6-uniform hypergraph of order $n$, then $\tau(H) \leqslant n / 4$.
\end{abstract}

Keywords: independence; clique; transversal 


\section{Introduction}

In this paper we study independence in graphs. Our main aim is to strengthen a result of Fajtlowicz $[3,4]$ on the independence of a graph given its maximum degree and maximum clique size. As a consequence of our result we give bounds on the independence number and transversal number of 6-uniform hypergraphs with maximum degree three.

A hypergraph $H$ consists of a finite vertex set $V(H)$ and a finite multiset $E(H)$ of edges, where each edge is a subset of $V(H)$. A hypergraph $H$ has rank $r$ if the largest size of an edge of $H$ is size $r$. A hypergraph $H$ is $k$-uniform if every edge of $H$ has size $k$. Every graph without loops is a 2-uniform hypergraph. The degree of a vertex $v$ in $H$, denoted by $d_{H}(v)$ or simply by $d(v)$ if $H$ is clear from context, is the number of edges of $H$ that contain $v$. The maximum degree among the vertices of $H$ is denoted by $\Delta(H)$. Two edges in $H$ are overlapping if they intersect in at least two vertices.

Two vertices $x$ and $y$ of $H$ are adjacent if some edge of $H$ contains both $x$ and $y$. A set $X$ of vertices in $H$ is a clique if every two vertices of $X$ are adjacent in $H$. A $k$-clique is a clique in $H$ of size $k$. The clique number $\omega(H)$ is the size of a maximum clique in $H$.

The neighborhood of a vertex $v$ in $H$, denoted $N_{H}(v)$ or simply $N(v)$ if $H$ is clear from context, is the set of all vertices different from $v$ that are adjacent to $v$. Two vertices $x$ and $y$ of $H$ are connected if there is a sequence $v_{0}, \ldots, v_{k}$ of vertices of $H$ with $x=v_{0}$ and $y=v_{k}$ in which $v_{i-1}$ is adjacent to $v_{i}$ for $1 \leqslant i \leqslant k$. A connected hypergraph is a hypergraph in which every two vertices are connected. A maximal connected subhypergraph of $H$ is a component of $H$.

For a subset $X$ of vertices in a hypergraph $H$, let $H[X]$ denote the hypergraph induced by the vertices in $X$, in the sense that $V(H[X])=X$ and $E(H[X])=\{e \cap X \mid e \in$ $E(H)$ and $|e \cap X| \geqslant 1\}$; that is, $E(H[X])$ is obtained from $E(H)$ by shrinking edges $e \in E(H)$ that intersect $X$ to the edges $e \cap X$.

If $H$ denotes a hypergraph and $X$ denotes a subset of vertices in $H$, then $H-X$ denotes that hypergraph obtained from $H$ by removing the vertices $X$ from $H$, removing all hyperedges that intersect $X$, and removing all resulting isolated vertices, if any. When $X=\{x\}$, we simply denote $H-X$ by $H-x$. In the literature this is sometimes called strongly deleting the vertices in $X$.

A twin in $H$ is a pair of vertices that are intersected by exactly the same set of edges; that is, a pair of vertices $u$ and $v$ is a twin in $H$ if every edge that contains $u$ also contains $v$, and every edge that contains $v$ also contains $u$. The hypergraph $H$ is twin-free if it has no twin. Hence if $H$ is twin-free, then for every pair of vertices $u$ and $v$ in $H$, there exists an edge $e$ such that $|e \cap\{u, v\}|=1$.

A set $S$ of vertices in a hypergraph $H$ is strongly independent if no two vertices in $S$ belong to a common edge. The strong independence number of $H$, which we denote by $\alpha(H)$, is the maximum cardinality of a strongly independent set in $H$. A subset of vertices in a hypergraph $H$ is a weakly independent set if it contains no edge of $H$.

A subset $T$ of vertices in a hypergraph $H$ is a transversal (also called vertex cover or hitting set in many papers) if $T$ intersects every edge of $H$. Equivalently, a set of vertices $S$ is transversal in $H$ if and only if $V(H) \backslash S$ is a weakly independent set in $H$. The 
transversal number $\tau(H)$ of $H$ is the minimum size of a transversal in $H$. We note that, $n(H)=\tau(H)+\alpha(H)$. Transversals in hypergraphs are well studied in the literature (see, for example, $[1,2,6,7])$.

Let $G$ be a graph, and let $X$ and $Y$ be disjoint subsets of $V(G)$. The set $E(X, Y)$ is the set of all the edges $x y$, with $x \in X$ and $y \in Y$. For each vertex $u \in V(G)$ let $w_{G}(u)$ denote the size of a largest clique in $G$ containing $u$. We will omit the subscript when $G$ is clear from the context.

\section{Independence in Graphs}

We shall prove the following result. The proof we present is similar to that of Fajtlowicz [3]. However in our proof we carefully choose a maximum independent set $S$ in the graph $G$ such that the number of edges from $S$ to vertices outside $S$ is minimized. With this choice of $S$, we establish a property on the graph $G$ by considering the operation of replacing a vertex in $S$ with a vertex outside $S$ in order to get a smaller number of edges between $S$ and vertices outside $S$.

Theorem 1. If $G$ is a graph of order $n$ and $p$ is an integer, such that (A) below holds, then $\alpha(G) \geqslant 2 n / p$.

(A): For every clique $X$ in $G$ there exists a vertex $x \in X$, such that $d(x)<p-|X|$.

Proof. Let $G=(V, E)$ be a graph of order $n$ and let $p$ be an integer such that (A) is satisfied. Let $S$ be a maximum independent set in $G$, such that $|E(S, V \backslash S)|\left(=\sum_{s \in S} d(s)\right)$ is minimized. Let $\alpha_{i}(S)$ denote the number of vertices in $V \backslash S$ with exactly $i$ neighbors in $S$. Since $S$ is a maximum independent set we note that $\alpha_{0}(S)=0$ and therefore the following holds.

$$
n-|S|=\alpha_{1}(S)+\alpha_{2}(S)+\cdots+\alpha_{|S|}(S) .
$$

Furthermore counting the number of edges in $E(S, V \backslash S)$ we obtain the following.

$$
\sum_{s \in S} d(s)=\alpha_{1}(S)+2 \alpha_{2}(S)+3 \alpha_{3}(S)+\cdots+|S| \alpha_{|S|}(S)
$$

Multiplying Equation (1) by 2 and subtracting Equation (2) we obtain the following.

$$
2 n-2|S|-\sum_{s \in S} d(s)=\alpha_{1}(S)-\alpha_{3}(S)-2 \alpha_{4}(S)-\cdots-(|S|-2) \alpha_{|S|}(S) \leqslant \alpha_{1}(S) .
$$

For each vertex $s \in S$, let $Y_{s}$ be the set of all vertices in $V \backslash S$ adjacent to $s$ but to no other vertex of $S$, and so every vertex in $Y_{s}$ has no neighbor in $S \backslash\{s\}$. If $Y_{s}$ does not induce a clique, then let $y_{1}, y_{2} \in Y_{s}$ be non-adjacent vertices and note that $S \cup\left\{y_{1}, y_{2}\right\} \backslash\{s\}$ is an independent set in $G$ of size greater than $|S|$, a contradiction. Therefore, $Y_{s} \cup\{s\}$ induces a clique in $G$. 
Suppose that $d(s)+\left|Y_{s}\right|+1 \geqslant p$. If there is a vertex $y \in Y_{s}$ such that $d(y)<d(s)$, then $(S \cup\{y\}) \backslash\{s\}$ contradicts the minimality of $|E(S, V \backslash S)|$. Therefore, for all $y \in Y_{s} \cup\{s\}$ we have $d(y)+\left|Y_{s} \cup\{s\}\right| \geqslant d(s)+\left|Y_{s}\right|+1 \geqslant p$, a contradiction to (A). This implies that $d(s)+\left|Y_{s}\right|+1 \leqslant p-1$, as $d(s),\left|Y_{s}\right|$ and $p$ are all integers. We now obtain the following, by Inequality (3),

$$
2 n \leqslant \alpha_{1}(S)+\sum_{s \in S} d(s)+2|S|=\sum_{s \in S}\left(\left|Y_{s}\right|+d(s)+2\right) \leqslant|S| p
$$

implying that $\alpha(G)=|S| \geqslant 2 n / p$ as desired.

As an immediate consequence of Theorem 1 we can prove the following result due to Fajtlowicz [3] on the independence of graph given its maximum degree and maximum clique size. We remark that in [4], Fajtlowicz studies some classes of graphs that achieve equality in the bound of Theorem 2.

Corollary 2. ([3]) If $G$ is a graph of order $n$ containing no clique of size $q$, then $\alpha(G) \geqslant$ $2 n /(\Delta(G)+q)$.

Proof. Let $G$ be a graph of order $n$ containing no clique of size $q$ and let $p=\Delta(G)+q$. For every clique $X$ in $G$ and for all vertices $x \in X$, we have $d(x)<\Delta(G)+1 \leqslant \Delta(G)+q-|X|=$ $p-|X|$, and therefore condition (A) holds in Theorem 1. By Theorem 1 we have that $\alpha(G) \geqslant 2 n / p=2 n /(\Delta(G)+q)$.

\section{Independence in hypergraphs of rank at most 6}

In this section we apply our main result, namely Theorem 1, to 3-regular, 6-uniform hypergraphs as there is a very interesting conjecture for this case, namely the TuzaVestergaard Conjecture which we state later. The application illustrates the power of our main result. However we remark that Theorem 1 can be used in the cases where the regularity is less than the uniformity.

We will prove the following theorem. We remark that the twin-free condition in Theorem 3 is necessary, since otherwise the result is not true. Consider, for example, the Fano-plane, where each vertex gets duplicated. The resulting hypergraph, $H$, is a 3-regular 6-uniform hypergraph on $n=14$ vertices, with strong independence number $\alpha(H)=1<$ $2 n / 23$.

Theorem 3. If $H$ is a twin-free 3-regular hypergraph of order $n$ and rank at most 6 , then $\alpha(H) \geqslant 2 n / 23$.

Before giving a proof of Theorem 3 we need a number of preliminary results. Let $H$ be a hypergraph of rank at most 6 . For a set $X$ of vertices in the hypergraph $H$, let

$$
\theta_{X}(H)=\max |X \cap e \cap f|
$$


where the maximum is taken over all distinct edges $e$ and $f$ in $H$. Let $e_{1}$ and $e_{2}$ be two (fixed) edges in $H[X]$ such that $\theta_{X}(H)=\left|X \cap e_{1} \cap e_{2}\right|$ and let $Y$ and $Z$ be defined by

$$
Y=X \backslash\left(e_{1} \cup e_{2}\right) \quad \text { and } \quad Z=X \cap e_{1} \cap e_{2} .
$$

We note that, $\theta_{X}(H)=|Z|$. We proceed further with a series of five lemmas that will prove useful when proving our main result.

Lemma 4. Let $H$ be a 3-regular hypergraph of rank at most 6 and let $X$ be a clique of size at least 8 in $H$. Then the following hold.

(a) $|Z| \geqslant 2$.

(b) If $H$ is twin-free and $|Y| \geqslant 2$, then $|Z|=2$.

(c) If $H$ is twin-free, then $|Y| \leqslant 2$.

Proof. (a) Let $x \in X$ and let $f_{1}, f_{2}, f_{3}$ be the three edges incident with $x$ in $H[X]$. If any two of these edges overlap, then $|Z| \geqslant 2$, as desired. Hence we may assume that $f_{i} \cap f_{j}=\{x\}$ for $1 \leqslant i<j \leqslant 3$. Since $H$ has rank at most $6,\left|f_{i}\right| \leqslant 6$ for $1 \leqslant i \leqslant 3$. Renaming edges if necessary, we may assume that $\left|f_{1}\right| \geqslant\left|f_{2}\right| \geqslant\left|f_{3}\right|$. Since $|X| \geqslant 8$, we note that $\left|f_{1}\right| \geqslant 4$ and $\left|f_{2}\right| \geqslant 2$. Let $v \in f_{2} \backslash\{x\}$. Since $X$ is a clique, the vertex $v$ is adjacent to every vertex in $f_{1}$. However, $d_{H}(v)=3$ and the edge $f_{2}$ does not intersect $f_{1} \backslash\{x\}$. Hence one of the two remaining edges, $g_{1}$ say, containing $v$ in $H[X]$ must contain at least two vertices of $f_{1} \backslash\{x\}$. But then $\left|f_{1} \cap g_{1}\right| \geqslant 2$, and so $|Z| \geqslant 2$.

(b) Suppose to the contrary that $H$ is twin-free and $|Y| \geqslant 2$, but $|Z| \geqslant 3$. Let $\left\{z_{1}, z_{2}, z_{3}\right\} \subseteq Z$. For $i=1,2,3$, let $g_{i}$ be the edge in $H[X]$ containing $z_{i}$ that is different from $e_{1}$ and $e_{2}$. Since $H$ is twin-free, we note that $z_{i} \notin g_{j}$ for $1 \leqslant i, j \leqslant 3$ and $i \neq j$. Let $\left\{y_{1}, y_{2}\right\} \subset Y$. Since $X$ is a clique, every vertex in $Y$ is adjacent to every vertex in $Z$. Thus, $\left\{y_{1}, y_{2}\right\} \subset g_{i}$ for $i=1,2,3$. But then $y_{1}$ and $y_{2}$ are twins, a contradiction. Therefore, $|Z| \leqslant 2$. By part (a), $|Z| \geqslant 2$. Consequently, $|Z|=2$.

(c) Suppose to the contrary that $H$ is twin-free, but $|Y| \geqslant 3$. By Part (b), $|Z|=2$. Let $Z_{1}=\left\{z_{1}, z_{2}\right\}$. For $i=1,2$, let $g_{i}$ be the edge in $H[X]$ containing $z_{i}$ that is different from $e_{1}$ and $e_{2}$. Since $H$ is twin-free, we note that $z_{1} \notin g_{2}$ and $z_{2} \notin g_{1}$. Since $X$ is a clique, every vertex in $Y$ is adjacent to every vertex in $Z$. Thus, $Y \subset g_{i}$ for $i=1,2$. But then $|Z|=\theta_{X}(H) \geqslant\left|X \cap g_{1} \cap g_{2}\right| \geqslant|Y| \geqslant 3$, contradicting Part (b).

Lemma 5. If $H$ is a twin-free 3-regular hypergraph of rank at most 6 , then $\omega(H) \leqslant 10$.

Proof. Suppose to the contrary that $\omega(H) \geqslant 11$. Let $X$ be a clique of size 11 in $H$, and let $H[X], \theta_{X}(H), e_{1}, e_{2}, Y$ and $Z$ be as defined earlier. Then, $11=|X|=\left|e_{1} \cup e_{2}\right|+|Y|=$ $\left|e_{1}\right|+\left|e_{2}\right|-|Z|+|Y|$. By Lemma $4(\mathrm{a}),|Z|=\theta_{X}(H) \geqslant 2$. Since $H$ has rank at most 6 , $\left|e_{1}\right|+\left|e_{2}\right| \leqslant 6+6=12$. If $|Z| \geqslant 3$, then $11 \leqslant 12-3+|Y|$, and so $|Y| \geqslant 2$, contradicting Lemma 4(b). Therefore, $|Z|=2$, implying that $11 \leqslant 12-2+|Y|$, or, equivalently, $|Y| \geqslant 1$.

Let $y \in Y$ and let $Z=\left\{z_{1}, z_{2}\right\}$. For $i=1,2$, let $g_{i}$ be the edge in $H[X]$ containing $z_{i}$ that is different from $e_{1}$ and $e_{2}$. Since $H$ is twin-free, we note that $z_{1} \notin g_{2}$ and $z_{2} \notin g_{1}$. Further since $X$ is a clique, we have that $y \in g_{1}$ and $y \in g_{2}$. Let $g_{3}$ denote the remaining edge containing $y$ in $H[X]$. 
For $i=1,2$, let $e_{i}^{\prime}=e_{i} \backslash Z$. Renaming the edges $e_{1}$ and $e_{2}$, if necessary, we may assume that $\left|e_{1}^{\prime}\right| \geqslant\left|e_{2}^{\prime}\right|$. If $\left|e_{1}^{\prime}\right| \leqslant 3$, then $\left|e_{1} \cup e_{2}\right| \leqslant 8$, implying that $|Y| \geqslant 3$, contradicting Lemma 4(c). Hence, $\left|e_{1}^{\prime}\right| \geqslant 4$. However, $\left|e_{1}^{\prime}\right|=\left|e_{1}\right|-|Z| \leqslant 6-2=4$. Consequently, $\left|e_{1}^{\prime}\right|=4$. We note therefore that either $|Y|=1$, in which case $\left|e_{2}^{\prime}\right|=4$, or $|Y|=2$, in which case $\left|e_{2}^{\prime}\right|=3$. Hence, $\left|e_{2}^{\prime}\right| \geqslant 3$.

If neither the edge $g_{1}$ nor the edge $g_{2}$ intersects $e_{2}^{\prime}$, then $e_{2}^{\prime} \subset g_{3}$. But then $\theta_{X}(H) \geqslant$ $\left|e_{2} \cap g_{3}\right| \geqslant\left|e_{2}^{\prime}\right| \geqslant 3$, a contradiction. Therefore renaming the vertices $z_{1}$ and $z_{2}$, if necessary, we may assume that $g_{1}$ intersects $e_{2}^{\prime}$. Let $w \in e_{2}^{\prime} \cap g_{1}$. Since $2=\theta_{X}(H) \geqslant\left|e_{1} \cap g_{1}\right|$, we note that the edge $g_{1}$ contains $z_{1}$ and at most one vertex of $e_{1}^{\prime}$. But then the edge, $e_{w}$ say, that contains $w$ and is different from $e_{2}$ and $g_{1}$, contains at least three vertices of $e_{1}^{\prime}$, implying that $\theta_{X}(H) \geqslant\left|e_{1} \cap e_{w}\right| \geqslant\left|e_{1}^{\prime}\right|-1=3$, a contradiction.

Lemma 6. If $H$ is a twin-free 3-regular hypergraph of rank at most 6 and $X$ is a 10-clique in $H$, then there exists a vertex $x \in X$ with $|N(x)| \leqslant 12$.

Proof. Let $X$ be a 10-clique in $H$, and let $H[X], \theta_{X}(H), e_{1}, e_{2}, Y$ and $Z$ be as defined earlier. Then, $10=|X|=\left|e_{1} \cup e_{2}\right|+|Y|=\left|e_{1}\right|+\left|e_{2}\right|-|Z|+|Y|$. By Lemma $4,|Y| \leqslant 2$ and $|Z|=\theta_{X}(H) \geqslant 2$.

We first consider the case when $|Z| \geqslant 3$. By Lemma 4 we note that $|Y| \leqslant 1$. Since $H$ has rank at most $6,10=\left|e_{1}\right|+\left|e_{2}\right|-|Z|+|Y| \leqslant 12-3+1=10$. Since we must have equality throughout this inequality chain, this implies that $|Z|=3$ and $|Y|=1$. Let $Z=\left\{z_{1}, z_{2}, z_{3}\right\}$ and for $i=1,2,3$, let $g_{i}$ be the edge in $H[X]$ containing $z_{i}$ that is different from $e_{1}$ and $e_{2}$. Since $H$ is twin-free, we note that $z_{i} \notin g_{j}$ for $1 \leqslant i, j \leqslant 3$ and $i \neq j$. Suppose that $g_{i}$ contains a vertex from $\left(e_{1} \cup e_{2}\right) \backslash Z$ for some $i, 1 \leqslant i \leqslant 3$. Then there are at least three vertices that belong to overlapping edges with $z_{i}$, implying that $\left|N_{H}\left(z_{i}\right)\right| \leqslant 12$ and the desired result follows. Hence we may assume that no vertex from $g_{i}$ belongs to $\left(e_{1} \cup e_{2}\right) \backslash Z$ for $i=1,2,3$. Since $X$ is a clique, we note that $y \in g_{i}$ for $i=1,2,3$. However this implies that $y$ is not adjacent to any vertex in $\left(e_{1} \cup e_{2}\right) \backslash Z$, a contradiction. Therefore, $|Z|=2$.

As before, let $Z=\left\{z_{1}, z_{2}\right\}$ and for $i=1,2$, let $g_{i}$ be the edge in $H[X]$ containing $z_{i}$ that is different from $e_{1}$ and $e_{2}$. Let $W=\left(e_{1} \cup e_{2}\right) \backslash Z$. Then, $10=|X|=|W|+|Y|+|Z|$, which as $|Z|=2$ and $0 \leqslant|Y| \leqslant 2$ implies that $6 \leqslant|W| \leqslant 8$. If $\left|g_{1} \cap W\right| \geqslant 2$, then $\left|N_{H}\left(z_{1}\right)\right| \leqslant 15-3=12$, and we are done. Hence we may assume that $\left|g_{1} \cap W\right| \leqslant 1$. Analogously, we may assume that $\left|g_{2} \cap W\right| \leqslant 1$. Let $W^{\prime}$ be the vertices in $W$ not covered by $g_{1} \cup g_{2}$. Let $W_{1}^{\prime}=W^{\prime} \cap e_{1}$ and let $W_{2}^{\prime}=W^{\prime} \cap e_{2}$, and so $\left|W^{\prime}\right|=\left|W_{1}^{\prime}\right|+\left|W_{2}^{\prime}\right|$.

Suppose that $|Y|=2$ and let $Y=\left\{y_{1}, y_{2}\right\}$. Then, $|W|=6$. Since $\left|g_{1} \cap W\right| \leqslant 1$ and $\left|g_{2} \cap W\right| \leqslant 1$, we note that $\left|W^{\prime}\right| \geqslant 4$. For $i=1,2$, let $f_{i}$ be the edge containing $y_{i}$ that is different from $g_{1}$ and $g_{2}$. Since $X$ is a clique, we have that $W^{\prime} \subset f_{i}$ for $i=1,2$. On the one hand, if $f_{1}=f_{2}$, then $\left\{y_{1}, y_{2}\right\}$ are twins. On the other hand, if $f_{1} \neq f_{2}$, then $\theta_{X}(H) \geqslant\left|f_{1} \cap f_{2} \cap W^{\prime}\right| \geqslant\left|W^{\prime}\right| \geqslant 4$. Both cases produce a contradiction. Hence, $|Y| \leqslant 1$.

Suppose that $|Y|=1$ and let $Y=\{y\}$. Then, $|W|=7$. Since $\left|g_{1} \cap W\right| \leqslant 1$ and $\left|g_{2} \cap W\right| \leqslant 1$, we note that $\left|W^{\prime}\right| \geqslant 5$. Let $g_{3}$ be the edge of $H[X]$ containing $y$ that is different from $g_{1}$ and $g_{2}$. Since $X$ is a clique, we have that $W^{\prime} \subset g_{3}$. Renaming $z_{1}$ and $z_{2}$ if 
necessary, we may assume that $\left|W_{1}^{\prime}\right| \geqslant\left|W_{2}^{\prime}\right|$, implying that $\theta_{X}(H) \geqslant\left|e_{1} \cap g_{3}\right| \geqslant\left|W_{1}^{\prime}\right| \geqslant 3$, a contradiction. Hence, $Y=\emptyset$.

Since $|Y|=0$, we have that $X=e_{1} \cup e_{2}$. Further since $H$ has rank at most 6 , this implies that $\left|e_{1}\right|=\left|e_{2}\right|=6$. For $i=1,2$, let $e_{i}^{\prime}=e_{i} \backslash Z$, and so $\left|e_{i}^{\prime}\right|=4$. Let $v$ be an arbitrary vertex in $X \backslash Z$. Renaming $z_{1}$ and $z_{2}$ if necessary, we may assume that $v \in e_{1}^{\prime}$. Let $e_{v}^{\prime}$ and $e_{v}^{\prime \prime}$ be the two edges in $H[X]$ different from $e_{1}$ that contain $v$. Since $X$ is a clique, the vertex $v$ is adjacent to every vertex in $e_{2}$. If $\left|e_{v}^{\prime} \cap e_{2}^{\prime}\right| \leqslant 1$, then $\left|e_{v}^{\prime \prime} \cap e_{2}^{\prime}\right| \geqslant 3$, implying that $\theta_{X}(H) \geqslant\left|e_{v}^{\prime \prime} \cap e_{2}\right| \geqslant 3$, a contradiction. Hence, $\left|e_{v}^{\prime} \cap e_{2}^{\prime}\right| \geqslant 2$. If $\left|e_{v}^{\prime} \cap e_{2}^{\prime}\right|>2$, then $\theta_{X}(H) \geqslant 3$, a contradiction. Hence, $\left|e_{v}^{\prime} \cap e_{2}^{\prime}\right|=2$. Analogously, $\left|e_{v}^{\prime \prime} \cap e_{2}^{\prime}\right|=2$. Since $v$ is adjacent to every vertex in $e_{2}$, we note that $\left(e_{v}^{\prime} \cap e_{2}^{\prime}\right) \cap\left(e_{v}^{\prime \prime} \cap e_{2}^{\prime}\right)=\emptyset$. This is true for every vertex $v \in X \backslash Z$. Hence every edge in $H[X]$ different from $e_{1}$ and $e_{2}$ has size 4 in $H[X]$ and contains two vertices in $e_{1}^{\prime}$ and two vertices in $e_{2}^{\prime}$.

Let $e_{1}^{\prime}=\left\{a_{1}, b_{1}, c_{1}, d_{1}\right\}$ and let $e_{2}^{\prime}=\left\{a_{2}, b_{2}, c_{2}, d_{2}\right\}$. Let $h_{1}$ be an arbitrary edge in $E(H[X]) \backslash\left\{e_{1}, e_{2}\right\}$. Renaming vertices if necessary, we may assume that $h_{1}=\left\{a_{1}, b_{1}, a_{2}, b_{2}\right\}$. Let $h_{2}$ and $h_{3}$ be the edges of $H[X]$ containing $a_{1}$ and $b_{1}$, respectively, that are different from $e_{1}$ and $h_{1}$. Then, $\left\{a_{1}, c_{2}, d_{2}\right\} \subset h_{2}$ and $\left\{b_{1}, c_{2}, d_{2}\right\} \subset h_{3}$. If $h_{2}=h_{3}$, then $a_{1}$ and $b_{1}$ are twins in $H$. If $h_{2} \neq h_{3}$, then $c_{2}$ and $d_{2}$ are twins in $H$. In both cases we contradict the fact that $H$ is twin-free.

Lemma 7. If $H$ is a 3-regular hypergraph of rank at most 6 and $X$ is a 9-clique in $H$, then there exists a vertex $x \in X$ with $|N(x)| \leqslant 13$.

Proof. Let $X$ be a 9-clique in $H$. If there are two twins in $H[X]$, then each of them have degree at most 13 and we are done. Hence we may assume that there are no twins in $H[X]$. For each edge, $f$, in $H$ containing some vertex $y$ we note that there are at most five vertices in $f \backslash\{y\}$ since $H$ has rank at most 6 . Define a graph $G_{X}$ with vertex set $X$ and with an edge between $x, x^{\prime} \in X$ if and only if $\left\{x, x^{\prime}\right\}$ is a subset of two distinct edges in $H$. Thus every neighbor, $x^{\prime}$, of a vertex $x \in X$ belongs to two edges of $H$ that contain both $x$ and $x^{\prime}$. This implies that $\left|N_{H}(x)\right| \leqslant 3 \times 5-d_{G_{X}}(x)$. Thus if $d_{G_{X}}(x) \geqslant 2$, then $\left|N_{H}(x)\right| \leqslant 13$, and we are done. Therefore we may assume that $d_{G_{X}}(x) \leqslant 1$ for all $x \in X$. Since $|X|=9$ is odd, this implies that some vertex $x \in X$ is an isolated vertex in $G_{X}$. Let $f_{1}, f_{2}, f_{3}$ be the three edges in $H[X]$ containing $x$.

Suppose that $\left|f_{1}\right|=6$. Let $v \in X \backslash f_{1}$. Renaming the edges $f_{2}$ and $f_{3}$, if necessary, we may assume that $v \in f_{2}$. Since $X$ is a clique, the vertex $v$ is adjacent to all five vertices in $f_{1} \backslash\{x\}$. Hence one of the two edges different from $f_{2}$ that contain $v$ must intersect $f_{1} \backslash\{x\}$ in at least three vertices. But this implies that $d_{G_{X}}(w) \geqslant 2$ for some $w \in f_{1}$, a contradiction. Therefore, $\left|f_{1}\right| \neq 6$. Analogously, $\left|f_{2}\right| \neq 6$ and $\left|f_{3}\right| \neq 6$.

Suppose that $\left|f_{1}\right|=4$. Let $U=X \backslash f_{1}$ and note that $|U|=5$. Let $u \in U$. Since $X$ is a clique, $u$ is adjacent to all three vertices in $f_{1} \backslash\{x\}$. Hence one of the two edges in $H[X]$ containing $u$ that is different from $f_{2}$ and $f_{3}$ contains at least two vertices in $f_{1} \backslash\{x\}$. Let $g_{u}$ be such an edge of $H[X]$ that contains $u$. If the five such edges, $g_{u}$, for all $u \in U$ are identical, then $\left|g_{u}\right| \geqslant|U|+2=7$, a contradicting the rank of $H$. Therefore there exist two distinct vertices $u$ and $u^{\prime}$ in $U$ such that $g_{u} \neq g_{u^{\prime}}$. Since both $g_{u}$ and $g_{u^{\prime}}$ contain at least two vertices in $f_{1} \backslash\{x\}$, and since $\left|f_{1} \backslash\{x\}\right|=3$, there exists some vertex in $f_{1} \backslash\{x\}$ 
that belongs to both $g_{u}$ and $g_{u^{\prime}}$. If $\left|g_{u} \cap g_{u^{\prime}} \cap f_{1}\right| \geqslant 2$, then there exists two twins in $H[X]$, a contradiction. Therefore, $g_{u} \cap g_{u^{\prime}} \cap f_{1}=\{w\}$ for some vertex $w \in f_{1} \backslash\{x\}$. But then $f_{1} \backslash\{x, w\} \subseteq N_{G_{X}}(w)$, implying that $d_{G_{X}}(w) \geqslant 2$, a contradiction. Therefore, $\left|f_{1}\right| \neq 4$. Analogously, $\left|f_{2}\right| \neq 4$ and $\left|f_{3}\right| \neq 4$.

Renaming the edges if necessary, we may assume that $\left|f_{1}\right| \geqslant\left|f_{2}\right| \geqslant\left|f_{3}\right|$. If $\left|f_{1}\right| \leqslant 3$, then $|X| \leqslant 7$, a contradiction. Hence, $\left|f_{1}\right| \geqslant 4$. However as shown earlier, $\left|f_{1}\right| \neq 4$ and $\left|f_{1}\right| \neq 6$. Therefore, $\left|f_{1}\right|=5$ and $\left|f_{2}\right| \geqslant 3$. Let $f_{1}^{\prime}=f_{1} \backslash\{x\}$ and note that $\left|f_{1}^{\prime}\right|=4$. Further, let $Q=\left\{q_{1}, q_{2}, q_{3}, q_{4}\right\}=X \backslash f_{1}$. Consider the vertex $q_{i} \in Q$ where $1 \leqslant i \leqslant 4$. Since $X$ is a clique, $q_{i}$ is adjacent to all four vertices in $f_{1}^{\prime}$. Further since no two edges of $H$ intersect in more than two vertices, this implies that there exists two edges $r_{i}$ and $r_{i}^{\prime}$ containing $q_{i}$, such that $\left|r_{i} \cap f_{1}^{\prime}\right|=\left|r_{i}^{\prime} \cap f_{1}^{\prime}\right|=2$ and $r_{i} \cap r_{i} \cap f_{1}^{\prime}=\emptyset$. Let there be $j$ distinct edges in

$$
E^{*}=\bigcup_{i=1}^{4}\left\{r_{i}, r_{i}^{\prime}\right\} .
$$

If $j=2$, then since $\left|f_{2}\right| \geqslant 3$ there are two twins in $f_{2} \backslash\{x\}$, a contradiction. Hence, $j \geqslant 3$. If two distinct edges in $E^{*}$ intersect in the same set of two vertices in $f_{1}^{\prime}$, then there are two twins in $f_{1}$, a contradiction. Hence every two distinct edges in $E^{*}$ have a different intersection in $f_{1}^{\prime}$. Since $j \geqslant 3$, there will therefore be at least three edges with both ends in $f_{1}^{\prime}$. This implies that $d_{G_{X}}(w) \geqslant 2$ for some $w \in f_{1}^{\prime}$, a contradiction.

Lemma 8. If $H$ is a 3-regular hypergraph of rank at most 6 and $X$ is a 8-clique in $H$, then there exists a vertex $x \in X$ with $|N(x)| \leqslant 14$.

Proof. Let $X$ be a 8-clique in $H$. By Lemma 4(a), $\theta_{X}(H) \geqslant 2$. Let $e_{1}$ and $e_{2}$ be two overlapping edges in $H[X]$ and let $x \in e_{1} \cap e_{2}$. Then, $|N(x)| \leqslant 14$.

We are now in a position to prove Theorem 3. Recall its statement.

Theorem 3. If $H$ is a twin-free 3-regular hypergraph of order $n$ and rank at most 6 , then $\alpha(H) \geqslant 2 n / 23$.

Proof. Let $G$ be the graph with vertex set $V(G)=V(H)$ and where two vertices are adjacent in $G$ if and only if they are adjacent in $H$. Clearly, $\alpha(G)=\alpha(H)$. Since $H$ is 3 -regular of rank at most 6 , we note that $\Delta(G) \leqslant 15$. Let $p=23$. If $X$ is a clique of size at most 7 in $G$, then for each vertex $x \in X$ we have $d_{G}(x)=\left|N_{H}(x)\right| \leqslant 15<p-|X|$. If $X$ is a clique of size 8 in $G$ (and therefore in $H$ ), there exists an $x \in X$, such that $d_{G}(x)=\left|N_{H}(x)\right|<15=p-|X|$, by Lemma 8. If $X$ is a clique of size 9 in $G$ (and therefore in $H$ ) there exists an $x \in X$, such that $d_{G}(x)=\left|N_{H}(x)\right|<14=p-|X|$, by Lemma 7. If $X$ is a clique of size 10 in $G$ (and therefore in $H$ ) there exists an $x \in X$, such that $d_{G}(x)=\left|N_{H}(x)\right|<13=p-|X|$, by Lemma 6 . Furthermore there is no clique in $G$ (or $H$ ) of size greater than 10 by Lemma 5. Therefore condition (A) holds in Theorem 1, implying that $\alpha(H)=\alpha(G) \geqslant 2|V(G)| / p=2|V(H)| / 23=2 n / 23$, by Theorem 1 .

We conjecture that the following holds. 
Conjecture 9. If $H$ is a twin-free 3-regular hypergraph of order $n$ and rank at most 6 , then $\alpha(H) \geqslant n / 10$.

We remark that if Conjecture 9 is true, then the bound is tight due to the following example. Let $H_{10}$ be the 6-uniform hypergraph with five edges, $e_{1}, e_{2}, e_{3}, e_{4}, e_{5}$, and ten vertices defined by $V\left(H_{10}\right)=\left\{u_{i, j, k} \mid 1 \leqslant i<j<k \leqslant 5\right\}$, where the vertex $u_{i, j, k}$ belongs to edges $e_{i}, e_{j}$ and $e_{k}$. Then, $H_{10}$ is 3-regular and 6-uniform. Furthermore, $H_{10}$ is twin-free as different vertices belong to different sets of edges. Further, for distinct vertices $u_{i, j, k}$ and $u_{i^{\prime}, j^{\prime}, k^{\prime}}$ in $H_{10}$, we note that $\{i, j, k\} \cap\left\{i^{\prime}, j^{\prime}, k^{\prime}\right\} \neq \emptyset$ as all indices cannot be distinct since they are between 1 and 5, implying that $u_{i, j, k}$ and $u_{i^{\prime}, j^{\prime}, k^{\prime}}$ are adjacent. Hence, $\alpha\left(H_{10}\right)=1=n / 10$, where $n=n\left(H_{10}\right)$. Therefore, $H_{10}$ would show that Conjecture 9 would be best possible.

\section{Transversals in 6-uniform hypergraphs}

Chvátal and McDiarmid [1] established the following upper bound on the transversal number of a uniform hypergraph in terms of its order and size.

Chvátal-McDiarmid Theorem. For $k \geqslant 2$, if $H$ is a $k$-uniform hypergraph of order $n$ and size $m$, then

$$
\tau(H) \leqslant \frac{n+\left\lfloor\frac{k}{2}\right\rfloor m}{\left\lfloor\frac{3 k}{2}\right\rfloor} .
$$

Let $n_{i}(H)$ denote the number of vertices in $H$ of degree $i$. As a consequence of the Chvátal-McDiarmid Theorem, we have the following two results.

Corollary 10. If $H$ is a 6-uniform hypergraph with $\Delta(H) \leqslant 3$, then

$$
18 \tau(H) \leqslant 3 n_{1}(H)+4 n_{2}(H)+5 n_{3}(H) .
$$

Proof. Let $H$ be a 6 -uniform hypergraph of order $n$ and size $m$ satisfying $\Delta(H) \leqslant 3$. For notational simplicity, let $n_{i}=n_{i}(H)$ for $i \in\{1,2,3\}$. Applying the Chvátal-McDiarmid Theorem to the hypergraph $H$, we have that

$$
\tau(H) \leqslant \frac{n+3 m}{9}=\frac{2 n+6 m}{18}=\frac{2\left(n_{1}+n_{2}+n_{3}\right)+\left(n_{1}+2 n_{2}+3 n_{3}\right)}{18}=\frac{3 n_{1}+4 n_{2}+5 n_{3}}{18},
$$

or, equivalently, $18 \tau(H) \leqslant 3 n_{1}(H)+4 n_{2}(H)+5 n_{3}$.

Corollary 11. If $H$ is a 3-regular 6-uniform hypergraph of order $n$, then $\tau(H) \leqslant 5 n / 18$. 
Our aim in this section is to lower the best known upper bound on the transversal number of a 3-regular 6 -uniform hypergraph of order $n$ from $5 n / 18 \approx 0.27777777 n$ (see Corollary 11 ) to $37 n / 138 \approx 0.268115942 n$. In order to state our result, let

$$
c_{1}=\frac{1}{6}, \quad c_{2}=\frac{2}{9}, \text { and } c_{3}=\frac{37}{138} .
$$

We first prove the following result on 6-uniform hypergraphs. We remark that if we allow edges of size less than 6 , then the result of Theorem 12 is not true anymore. For example, 3-regular 3-uniform hypergraphs on $n$ vertices may have transversal number $n / 2$ (see, [5]).

Theorem 12. If $H$ is a 6-uniform hypergraph with $\Delta(H) \leqslant 3$, then

$$
\tau(H) \leqslant c_{1} n_{1}(H)+c_{2} n_{2}(H)+c_{3} n_{3}(H) .
$$

Proof. We proceed by induction on the order of a 6-uniform hypergraph $H$ satisfying $\Delta(H) \leqslant 3$. For a hypergraph $H^{\prime}$ with $\Delta\left(H^{\prime}\right) \leqslant 3$, let

$$
\theta\left(H^{\prime}\right)=c_{1} n_{1}\left(H^{\prime}\right)+c_{2} n_{2}\left(H^{\prime}\right)+c_{3} n_{3}\left(H^{\prime}\right) .
$$

Hence we wish to show that $\tau(H) \leqslant \theta(H)$. If $m(H)=0$, then $\tau(H)=0$ and the result is immediate. Hence we may assume that $m(H) \geqslant 1$, implying that $|V(H)| \geqslant 6$. If $|V(H)|=6$, then $\tau(H)=1 \leqslant \theta(H)$. This establishes the base cases when $|V(H)| \leqslant 6$. Let $H$ be a 6 -uniform hypergraph such that $\Delta(H) \leqslant 3$ and assume the theorem holds for all 6-uniform hypergraphs $H^{\prime}$ satisfying $\Delta\left(H^{\prime}\right) \leqslant 3$ and $n\left(H^{\prime}\right)<n(H)$.

If $\Delta(H) \leqslant 2$, then $n_{3}(H)=0$ and the theorem holds by Corollary 10 of the ChvátalMcDiarmid Theorem. Hence we may assume that $\Delta(H)=3$. We consider two cases, depending on whether $H$ has twins of degree 3 and or not.

Suppose first that $H$ contains two twins, $x_{1}$ and $x_{2}$, of degree 3 . Let $X=\left\{x_{1}, x_{2}\right\}$ and let $H^{\prime}=H-\left\{x_{1}, x_{2}\right\}$. Thus, $H^{\prime}$ is obtained from $H$ by removing the vertices $X$ from $H$ removing the three hyperedges that intersect $X$, and removing all resulting isolated vertices, if any. Let $T^{\prime}$ be a minimum transversal in $H^{\prime}$. Then, $T=T^{\prime} \cup\left\{x_{1}\right\}$ is a transversal in $H$, and so $\tau(H) \leqslant|T|=\left|T^{\prime}\right|+1=\tau\left(H^{\prime}\right)+1$. We note that by removing the three edges that contain $X$, the degrees of $x_{1}$ and $x_{2}$ drop from 3 to zero. Further, if some vertex $v \notin X$ belongs to $i$ of the deleted edges its degree drops to $d_{H}(v)-i$ in $H^{\prime}$, implying that the sum of the degrees of vertices not in $X$ decrease by 12 in $H^{\prime}$ due to the 6-uniformity of $H$. If the degree of a vertex drops from 1 to 0 in $H^{\prime}$, then it decreases $\theta(H)$ by $c_{1}$. If its degree drops from 2 to 1 in $H^{\prime}$, then it decreases $\theta(H)$ by $c_{2}-c_{1}$, while if its drops from 3 to 2 in $H^{\prime}$, then it decreases $\theta(H)$ by $c_{3}-c_{2}$. Since $c_{1} \geqslant c_{2}-c_{1} \geqslant c_{3}-c_{2}$, we therefore have that whenever the degree of a vertex drops by 1 in $H^{\prime}$, then it decreases $\theta(H)$ by at least $c_{3}-c_{2}$. Therefore,

$$
\theta\left(H^{\prime}\right) \leqslant \theta(H)-2 c_{3}-12\left(c_{3}-c_{2}\right)=\theta(H)+12 c_{2}-14 c_{3}<\theta(H)-1,
$$

implying that

$$
\tau(H) \leqslant|T|=\left|T^{\prime}\right|+1 \leqslant \theta\left(H^{\prime}\right)+1<\theta(H)
$$


Hence if $H$ contains two twins, $x_{1}$ and $x_{2}$, of degree 3, then $\tau(H)<\theta(H)$. We may therefore assume $H$ has no twins of degree 3, for otherwise the desired result holds.

Recall that by our earlier assumption, $\Delta(H)=3$. Let $R$ contain all vertices in $H$ of degree 3. Then, $H[R]$ is a 3-regular hypergraph of rank at most six and with no twins. By Theorem 3 there exists a strongly independent set, $I$, in $H[R]$ of size at least $2|R| / 23$. Let $H^{\prime}=H-I$ and let $E^{*}=\left\{e_{1}^{*}, e_{2}^{*}, \ldots, e_{3|I|}^{*}\right\}$ be the set of $3|I|$ edges containing vertices from $I$. As observed earlier, when we delete an edge $e$ from a 6-uniform hypergraph $H$ with maximum degree at most 3 and if $v \in e$, then $\theta(H)$ drops by $c_{3}-c_{2}$ if $d_{H}(v)=3$, $\theta(H)$ drops by $c_{2}-c_{1}$ if $d_{H}(v)=2$, and $\theta(H)$ drops by $c_{1}$ if $d_{H}(v)=1$. Further, $c_{1} \geqslant c_{2}-c_{1} \geqslant c_{3}-c_{2}$. Thinking of $H^{\prime}$ as being obtained from $H$ by removing the edges $e_{1}^{*}, e_{2}^{*}, e_{3}^{*}, \ldots, e_{3|I|}^{*}$ in that order, we note that exactly $|R|$ times we $\operatorname{drop} \theta(H)$ by $c_{3}-c_{2}$, once for each vertex in $R$ (noting that each vertex in $R$ is contained in at least one edge in $\left.E^{*}\right)$. Further, at least $|I|$ times we drop $\theta(H)$ by $c_{1}$ since all edges are removed from the vertices in the independent set $I$. The total sum of the degrees of vertices decrease by $6\left|E^{*}\right|$ in $H^{\prime}$ due to the 6-uniformity of $H$. We therefore obtain the following.

$$
\begin{aligned}
\theta\left(H^{\prime}\right)+|I| & \leqslant|I|+\theta(H)-\left(c_{3}-c_{2}\right)|R|-c_{1}|I|-\left(c_{2}-c_{1}\right)\left(6\left|E^{*}\right|-|R|-|I|\right) \\
& =|I|+\theta(H)-\left(c_{3}-c_{2}\right)|R|-c_{1}|I|-\left(c_{2}-c_{1}\right)(18|I|-|R|-|I|) \\
& =\theta(H)-\left(c_{3}-c_{2}-c_{2}+c_{1}\right)|R|-\left(c_{1}+17 c_{2}-17 c_{1}-1\right)|I| \\
& =\theta(H)-\left(c_{3}-2 c_{2}+c_{1}\right)|R|-\left(17 c_{2}-16 c_{1}-1\right)|I| \\
& \leqslant \theta(H)-\left(c_{3}-2 c_{2}+c_{1}\right)|R|-2\left(17 c_{2}-16 c_{1}-1\right)|R| / 23 \\
& =\theta(H)-\left(23 c_{3}-46 c_{2}+23 c_{1}+34 c_{2}-32 c_{1}-2\right)|R| / 23 \\
& =\theta(H)-\left(23 c_{3}-12 c_{2}-9 c_{1}-2\right)|R| / 23 \\
& =\theta(H) .
\end{aligned}
$$

Applying the inductive hypothesis to $H^{\prime}$, we have that $\tau\left(H^{\prime}\right) \leqslant \theta\left(H^{\prime}\right)$. Every transversal in $H^{\prime}$ can be extended to a transversal in $H$ by adding to it the set $I$, implying that

$$
\tau(H) \leqslant \tau\left(H^{\prime}\right)+|I| \leqslant \theta\left(H^{\prime}\right)+|I| \leqslant \theta(H),
$$

which completes the proof.

As a consequence of Theorem 12, we have the following result for 6-uniform hypergraphs.

Corollary 13. If $H$ is a 3-regular 6 -uniform hypergraph of order $n$, then $\tau(H) \leqslant 37 n / 138 \approx$ $0.268115942 n$.

Proof. If $H$ is a 3-regular 6-uniform hypergraph of order $n$, then by Theorem 12 we have that $\tau(H) \leqslant c_{1} n_{1}(H)+c_{2} n_{2}(H)+c_{3} n_{3}(H)=0+0+37 n / 138$.

We remark that Corollary 13 gives support for the following long-standing conjecture due to Tuza and Vestergaard [8], in that it lowers the best known upper bound on the transversal number of a 3-regular 6-uniform hypergraph of order $n$ from $5 n / 18$ to $37 n / 138$.

Tuza-Vestergaard Conjecture. If $H$ is a 3-regular 6-uniform hypergraph of order $n$, then $\tau(H) \leqslant n / 4=0.25 n$. 


\section{Acknowledgements}

Research of Michael A. Henning supported in part by the South African National Research Foundation and the University of Johannesburg. Christian Löwenstein is indebted to the Baden-Württemberg Stiftung for the financial support of this research project by the Eliteprogramme for Postdocs.

\section{References}

[1] V. Chvátal and C. McDiarmid. Small transversals in hypergraphs. Combinatorica, 12:19-26, 1992.

[2] E. J. Cockayne, S. T. Hedetniemi and P. J. Slater. Matchings and transversals in hypergraphs, domination and independence-in trees. J. Combin. Theory B, 27:78-80, 1979.

[3] S. Fajtlowicz. On the size of independent sets in graphs. Congressus Numerantium, 21:269-274, 1978.

[4] S. Fajtlowicz. Independence, clique size and maximum degree. Combinatorica, 4:3538, 1984.

[5] M. A. Henning and A. Yeo. Hypergraphs with large transversal number and with edge sizes at least three. J. Graph Theory, 59:326-348, 2008.

[6] F. C. Lai and G. J. Chang. An upper bound for the transversal numbers of 4-uniform hypergraphs. J. Combin. Theory Ser. B, 50:129-133, 1990.

[7] S. Thomassé and A. Yeo. Total domination of graphs and small transversals of hypergraphs. Combinatorica, 27:473-487, 2007.

[8] Zs. Tuza and P. H. Vestergaard. Domination in partitioned graphs. Discussiones Math. Graph Theory, 22:199-210, 2002. 